\title{
REVIEW ON THE APPLICATION OF ECOSYSTEM MODELS IN BIODIVERSITY ANALYSES
}

\author{
*Imam, T.S., and Abdullahi, N. \\ Biological Sciences Department, Bayero University, Kano, PMB 3011, Kano, Nigeria. \\ Correspondence author: tsimam2001@yahoo.com
}

\begin{abstract}
This paper is an exposition with the sole aim of highlighting the relevance of ecosystem models in the analyses of biodiversity. The structure of ecosystem models enables researchers to design and consequently formulate monitoring programs that will be useful to the conservation of biodiversity. Ecosystem theoretical models discussed in the paper include Lotka and Volterra Predator-Prey Model; Constructal Law; Kleiber's Law; Metabolic Theory of Ecology; Occupancy-Abundance Relationship; Allometry Scaling; Rapoport's Rule; Ewin's Sampling Formula; Thorson's Rule; Rench's Rule, Unified Neutral Theory of Biodiversity (UNTB), and Ecosystem Forecasting. Lastly Biodiversity Action Plan as one of the applications of Ecosystem Theoretical models project was explained.
\end{abstract}

Keywords: action plan, biodiversity, ecosystem, models

\section{INTRODUCTION}

Ecosystem models are mathematical representations of ecosystems. Ecosystem models are a development of theoretical ecology which goal is to characterize major ecosystem dynamics in order to predict the behaviors of the systems. As a result of complexity of ecosystem, ecosystem models typically simplify the systems to a limited number of pragmatic components. These can be in form of analyzing a particular species abundance, composition etc or in form of studying species broad functional types such as autotrophs, heterotrophs or saprotrophs.

\section{Structure of Ecosystem Models}

The simplification process as described above clearly reduces an ecosystem to a small number of state variables. These can represent ecological components in terms of numbers of discrete individuals or quantify the component continuously as a measure of the total biomass of all organisms of that type, especially using model units (e.g. mass of carbon per unit area/ volume). Some ecological interactions are derived from biochemical processes (e.g. biogeochemical cycles). Typically relationships are computed statistically or heuristically (e.g. trial and error technique). After establishing the components to be modeled and the relationships between them, another fundamental factor in the ecosystem model structure is the representation of space used (e.g. study area and location). Ecosystem model should compose of modeled biological populations that experience growth, interact with other populations and suffer mortality (Wikipedia Encyclopedia, 2009).

There are numerous ecosystem models in use. However, there is a lot of similarity between them. This review therefore discusses models that are unique, based on their applications to biodiversity analysis for the purpose of formulation of its conservation and monitoring projects.

\section{Lotka and Volterra Predator-Prey Model}

This ecosystem model as the name implies is devised in order to explain the relationship between predator and prey organisms. It is one of the earliest and wellknown model, named after Alfred J.Lotka (1925) and Vito Volterra (1926) were the ones that devised it. Volterra initially produced the model for the purpose of explaining fluctuations in fish and shark populations observed in the Adriatic Sea after the First World War. Later the formula subsequently started to be applied to general predator-prey relationships (Volterra, 1926; Israel, 1988).

This model involves pair of differential equations, representing prey and its predator. Presented as follows:

$\mathrm{dX} / \mathrm{dt}=\mathrm{a} \cdot \mathrm{X}-$ B.X.Y; $\mathrm{dY} / \mathrm{dt}=\mathrm{Y} \cdot$ B.X.Y. $-\delta Y$

Where: $X=$ number/ concentration of the prey species; $Y=$ number/ concentration of the predator species; $a=$ prey species' growth rate; $B=$ predation rate of $Y$ upon $X ; Y=$ assimilation efficiency of $Y ; \delta=$ mortality rate of the predator species.

\section{Constructal Law}

The constructal law defines the time direction of all evolutionary design phenomena. It defines what it means to be "fittest", to "survive", and to be efficient. "Constructal" is a word coined by Adrian Bejan, ( Latin: construere, to construct) in order to designate the natural tendency of flow system (rivers, trees and branches etc) to morph in a constructal evolutionary process toward greater and greater flow access in time. If the flows stop, the system is dead (in thermodynamic equilibrium) (Bejan et al., 2008). The constructal law was stated by Bejan in 1996 as follows:

"For a finite-size system to persist in time (to live), it must evolve in such a way that it provides easier access to the imposed currents that flow through it." (Bejan and Lorento, 2010). The constructal law is the physics law of life and evolution. Application of constructal law in biodiversity analysis includes: 
- The distribution of tree sizes and numbers on the forest floor.

- The entire architecture of vegetation: roots, trunks, canopies, branches, leaves, and the forest, including the prediction of Leonardo da Vinci's rule, Huber's rule, and the Fibonacci sequence.

- The scaling law of all animal locomotion (running, flying, swimming): speeds, frequencies, forces and the work spent per unit of mass moved and distance traveled.

- Kleiber's law, the relationship between metabolic rate and body size

\section{Kleiber's Law}

Kleiber's Law (Kleiber, 1932), named after Max Kleiber's biological work in the early $1930 \mathrm{~s}$, refers to the observation that, for the vast majority of animals, an animal's metabolic rate scales to the $3 / 4$ power of the animal's mass. Symbolically: if $q_{0}$ is the animal's metabolic rate, and $M$ the animal's mass, then Kleiber's law states that $q_{0} \sim M^{3 / 4}$. Thus a cat, having a mass 100 times that of a mouse, will have a metabolism roughly 31 times greater than that of a mouse. In plants, the exponent is found to be close to 1 . Young (i.e., small) organisms respire more per unit of weight than old (large) ones of the same species because of the overhead costs of growth, but small adults of one species respire more per unit of weight than large adults of another species because a larger fraction of their body mass consists of structure rather than reserve; structural mass involves maintenance costs, reserve mass does not (Kleiber, 1947).

Attempts to understand the metabolic rate of a multicellular organism involves the product between average basal metabolic rate, and number of cells. In plants, the exponent of mass is found to be close to 1 . The data from study of oxygen consumption metabolic rates in cells in vitro suggests that the exponent is not only far less than $3 / 4$, but also becomes negative for things less than one gram in size (in case of microorganisms) (Kleiber, 1947).

\section{Metabolic Theory of Ecology}

The metabolic theory of ecology (MTE) is an extension of Kleiber's law and posits that the metabolic rate of organisms is the fundamental biological rate that governs most observed patterns in ecology (Brown et al., 2004).

MTE is based on an interpretation of the relationships between body size, body temperature, and metabolic rate across all organisms. Small-bodied organisms tend to have higher mass-specific metabolic rates than largerbodied organisms. Organisms that operate at warm temperatures through endothermy or by living in warm environments tend towards higher metabolic rates than organisms that operate at colder temperatures (Gilooly et al., 2005). This pattern is consistent from the unicellular level up to the level of the largest animals on the planet. The metabolic theory of ecology's main implication is that metabolic rate, and the influence of body size and temperature on metabolic rate, provides the fundamental constraints by which ecological processes are governed. Small animals tend to grow fast, breed early, and die young (Hutchinson and MacArthur, 1959). An organisms' metabolic rate determines its rate of food consumption, which in turn determines its rate of growth. It has been observed that there are more small species than large species. In addition, there are more species in the tropics than at higher latitudes. Classically, the latitudinal gradient in species diversity has been explained by factors such as higher productivity or reduced seasonality (Rohde, 1992).

MTE explains this pattern as being driven by the kinetic constraints imposed by temperature on metabolism. If a higher rate of molecular evolution causes increased speciation rates, then adaptation and ultimately speciation may occur more quickly in warm environments and in small bodied species, ultimately explaining observed patterns of diversity across body size and latitude (Savage et al., 2004).

At the ecosystem level, MTE explains the relationship between temperature and production of biomass. The average production to biomass ratio of organisms is higher in small organisms than large ones (Savage et al., 2007). As production consistently scales with body mass, MTE predicts that the primary factor that causes differing rates of production between ecosystems is temperature and not the mass of organisms within the ecosystem. This suggests that regions with similar climactic factors would sustain the same primary production, even if standing biomass is different (Reich et al., 2006).

\section{Occupancy-Abundance Relationship}

The occupancy-abundance $(O-A)$ relationship is the relationship between the abundance of species and the size of their ranges within a region. This relationship is perhaps one of the most well-documented relationships in macroecology, and applies both intra- and interspecifically (within and among species). In most cases, the O-A relationship is a positive relationship. Most evaluations of O-A relationships do not evaluate species over their entire (global) range, but document abundance and occupancy patterns within a specific region (Hui et al., 2009). It is believed that species decline in abundance and become more patchily distributed towards the margin of their range. In the same manner, an assemblage of species within the study region can be expected to contain some species near the core and some near the periphery of their ranges, leading to a positive interspecific O-A relationship

Many species exhibit density-dependent dispersal and habitat selection (Amarasekare, 2004). For species exhibiting this pattern, dispersal into what would otherwise be sub-optimal habitats can occur when local abundances are high in high quality habitats, thus increasing the size of the species geographic range. For example, Zuckerberg et al. (2009) have demonstrated that for breeding birds in New York, most species that underwent changes in abundance (positive or negative) between 1985 and 2005 showed concurrent changes in range size. Using a dipswitch test with 15 criteria, Hui et al. (2009) examined the ability of eight models of this kind to estimate the abundance of 610 southern African bird species. Models based on the scaling pattern of occupancy (i.e., those that reflect the scale dependence of species range size) produced the most reliable abundance estimates, and therefore are recommended for assemblage-scale regional abundance estimation (Hui et al., 2009). Intra- and Interspecific occupancyabundance relationships are important in the following ways:

1. Setting harvest rates - Especially in the case of (in fisheries) the proportion of the total population of a species expected to be captured at a given effort is expected to increase as range size decreases. 
2. Conservation biology - The existence of positive intraspecific O-A relationships would exacerbate the risks faced by imperiled species. Not only would reductions in range size and number of sites occupied directly increase the threat of extinction, but extinction risk would be further increased by the concurrent decline in abundance. 3. Biodiversity inventory - An interspecific O-A relationship implies that those species that have a restricted distribution (and hence will be important for conservation reasons) will also have low abundance within their range

4. Conservation -Species will not only be at risk of extinction due to low abundance, but because species with low abundance are expected to have restricted distributions, they are at risk of local catastrophe leading to global extinction. Also, because rare species tend to have restricted distributions; conservation programmes aimed at prioritizing sites for multi-species conservation will include fewer habitats for rare species than common species.

5. Invasive species -The logic relating positive $O-A$ relationships to invasion biology is the same as that relating O-A relationships to conservation concerns. Specifically, as an invading species increases in local abundance, its range can be expected to expand, further confounding control efforts.

\section{Allometry Scaling}

Allometry often studies shape differences in terms of ratios of the objects' dimensions. Two objects of different size but common shape will have their dimensions in the same ratio. Take, for example, a biological object that grows as it matures. Its size changes with age but the shapes are similar. Allometry is the study of the relationship between size and shape, first outlined by Otto Snell in 1892 and Julian Huxley in 1932. Allometry is a well-known study in biology for practical applications to the differential growth rates of the parts of a living organism's body. One application is in the study of various insect species (e.g., the Hercules Beetle), where a small change in overall body size can lead to an enormous and disproportionate increase in the dimensions of appendages such as legs, antennae, or horns (Fairbain, 1997). The relationship between the two measured quantities is often expressed as a power-law:

$$
\begin{aligned}
& y \propto x^{a} \text { or in a logarithmic form: } \\
& \log y \sim a \log x
\end{aligned}
$$

Where $a$ is the scaling exponent of the law. Methods for estimating this exponent from data are complicated because the usual method for fitting lines (least-squares regression) does not account for error variance in the independent variable (e.g., log body mass). Studies of ontogenetic allometry often use lizards or snakes as model organisms because they lack parental care after birth or hatching and because they exhibit a large range of body size between the juvenile and adult stage. Lizards often exhibit allometric changes during their ontogeny. Allometry also examines shape variation among individuals of a given age (and sex), which is referred to as static allometry. Comparisons of species are used to examine interspecific or evolutionary Allometry (Fairbain, 1997).
Allometric scaling is any change that deviates from isometry. A classic example is the skeleton of mammals, which becomes much more robust and massive relative to the size of the body as the body size increases. An isometrically scaling organism would see all volumebased properties change with mass to the first power (1.0), all surface area-based properties change with mass to the 2/3 power, and all length-based properties change with mass to the $1 / 3$ power (Kozlowski and Konarzewski, 2004).

\section{Rapoport's Rule}

Rapoport's rule is an ecological hypothesis that states that latitudinal ranges of plants and animals are generally smaller at low than at high latitudes. Stevens (1996) named the rule after Eduardo $\mathrm{H}$. Rapoport, who had earlier provided evidence for the phenomenon for subspecies of mammals (Rapoport,1982). Rapoport's rule states that latitudinal ranges of species are generally smaller at low than at high latitudes.

Stevens used the rule to "explain" greater species diversity in the tropics in the sense that latitudinal gradients in species diversity and the rule have identical exceptional data and so must have the same underlying cause. Narrower ranges in the tropics would facilitate more species to coexist. This rule was later extended to altitudinal gradients, claiming that altitudinal ranges are greatest at greater altitudes, and to depth gradients in the oceans. For example, marine teleost fishes have the greatest latitudinal ranges at low latitudes. In contrast, freshwater fishes do show the trend, although only above a latitude of about 40 degrees North (Stevens, 1996). Rohde (1996) explained the fact that the rule is restricted to very high latitudes by effects of glaciations which have wiped out species with narrow ranges. Another explanation of Rapoport's rule is the "climatic variability" or "seasonal variability hypothesis". According to this hypothesis, seasonal variability selects for greater climatic tolerances and therefore wider latitudinal ranges.

Most of these explanations can be excluded for the Monogenea, whose larvae are never planktotrophic, their larvae are always short-lived (Gusev, 1978). Gyrodactylidae are among the smallest Monogenea, and Monogenea do not possess calcareous skeletons (Mileikovsky, 1971). The conclusion is that the most likely explanation for the Monogenea (and by implication for other groups) is that small larvae cannot locate suitable habitats at low temperatures, where physiological, including sensory processes are slowed down, and/or that low temperatures prevent the production of sufficient numbers of pelagic larvae, which would be necessary to find suitable habitats in the vast oceanic spaces (Rohde, 1985; Gallardo and Penchaszadeh, 2001).

\section{Ewens's Sampling Formula}

In population genetics, Ewens' sampling formula, introduced by Warren Ewens (Ewens, 1972), states that under certain conditions, if a random sample of $n$ gametes is taken from a population and classified according to the gene at a particular locus then, the probability that there are $a_{1}$ alleles represented once in the sample, and $a_{2}$ alleles represented twice, and so on, is 


$$
\operatorname{Pr}\left(a_{1}, \ldots, a_{n}\right)=\frac{n !}{\theta(\theta+1) \cdots(\theta+n-1)} \prod_{j=1}^{n} \frac{\theta^{a_{j}}}{j^{a_{j}} a_{j} !},
$$

For some positive number $\theta_{\text {, whener }} a_{1}, \ldots, a_{n}$ is a sequence of nonnegative integers such that

$$
a_{1}+2 a_{2}+3 a_{3}+\cdots+n a_{n}=n \text {. }
$$

The phrase "under certain conditions", used above, must of course be made precise. The assumptions are (1) the sample size $n$ is small by comparison to the size of the whole population, and (2) the population is in statistical equilibrium under mutation and genetic drift and the role of selection at the locus in question is negligible, and (3) every mutant allele is novel (Ewens, 1972).

When $\theta=0$, the probability is 1 that all $n$ genes are the same. When $\theta=1$, then the distribution is precisely that of the integer partition induced by a uniformly distributed random permutation. As $\theta \rightarrow \infty$, the probability that no two of the $n$ genes are the same approaches 1 . This family of probability distributions enjoys the property that, if after the sample of $n$ is taken, $m$ of the $n$ gametes are chosen without replacement, then the resulting probability distribution on the set of all partitions of the smaller integer $m$ is just what the formula above would give if $m$ were put in place of $n$ (Ewens, 1972; Johnson et al., 1997).

\section{Thorson's rule}

Thorson's rule (named after Gunnar Thorson by Mileikovsky in 1971) states that (Thorson, 1957) benthic marine invertebrates at low latitudes tend to produce large numbers of eggs developing to pelagic (often planktotrophic) and widely-dispersing larvae, whereas at high latitudes such organisms tend to produce fewer and larger lecithotrophic (yolk-feeding) eggs and larger offspring, often by viviparity or ovoviviparity, which are often brooded (Krug, 1998). The rule was originally established for marine bottom invertebrates, but it also applies to a group of parasitic flatworms, monogenean ectoparasites on the gills of marine fish. Most low-latitude species of Monogenea produce large numbers of ciliated larvae. However, at high latitudes, species of the entirely

viviparous family Gyrodactylidae, which produce few nonciliated offspring and are very rare at low latitudes, represent the majority of gill Monogenea, i.e., about 80$90 \%$ of all species at high northern latitudes, and about one third of all species in Antarctic and sub-Antarctic waters, against less than $1 \%$ in tropical waters. A study in 2001 indicated that two factors are important for Thorson's rule to be valid for marine gastropods: 1 ) the habitat must include rocky substrates, because softbottom habitats appear to favour non-pelagic development; and 2) a diverse assemblage of taxa needs to be compared to avoid the problem of phyletic constraints, which could limit the evolution of different developmental modes (Gallardo and Penschaszardeh, 2001; Azaele et al., 2006).

\section{Rensch's Rule}

Rensch's rule is concerned with the relationship between the extent of sexual size dimorphism (SSD) and which sex is larger. Across species within a lineage, size dimorphism will increase with increasing body size when the male is the larger sex, and decrease with increasing average body size when the female is the larger sex. Examples of phylogenetic lineages that appear to follow this rule are primates, pinnipeds, and artiodactyls (Fairbairn, 1997).

\section{Stochastic Modeling of Species Abundances under the Unified Neutral Theory of Biodiversity (UNTB)}

UNTB distinguishes between a dispersal-limited local community of size $J$ and a so called metacommunity from which species can (re)immigrate. The expected number $S_{M}(n)$ of species in the metacommunity having exactly $n$ individuals is (Vallade and Houchmandzadeh, 2003)

$$
S_{M}(n)=\frac{\theta}{n} \frac{\Gamma\left(J_{M}+1\right) \Gamma\left(J_{M}+\theta-n\right)}{\Gamma\left(J_{M}+1-n\right) \Gamma\left(J_{M}+\theta\right)}
$$

where $\theta=\left(J_{M}-1\right) \nu /(1-\nu) \approx J_{M} \nu$ is called the fundamental biodiversity number. For large metacommunities and $n \ll J_{M}$.

$$
S_{M}(n) \approx \frac{\theta}{n}\left(\frac{\dot{J}_{M}}{J_{M}+\theta}\right)^{n}
$$

UNTB thus predicts that in dispersal limited communities rare species become even rarer. The log-normal distribution describes the maximum and the abundance of common species very well but underestimates the number of very rare species considerably which becomes only apparent for very large sample sizes (Hubbell, 2001).

The Unified Theory unifies biodiversity, as measured by species-abundance curves, with biogeography, as measured by species-area curves. Species-area relationships show the rate at which species diversity increases with area. The topic is of great interest

$$
S(\theta)=1+\theta \ln \left(1+\frac{J-1}{\theta}\right) \text {. }
$$

to conservation biologists in the design of reserves, as it is often desired to harbor as many species as possible. The most commonly encountered relationship is the power law given by

$$
S=C A^{z}
$$

Where $S$ is the number of species found, $A$ is the area sampled, and $c$ and $z$ are constants. This relationship, with different constants, has been found to fit a wide range of empirical data. The formula for species composition derived above may be used to calculate the expected number of species present in a community under the assumptions of the Unified Theory. In symbols 
This formulation is predicated on a random placement of individuals. The population of any species is represented

$$
\dot{x}=b-x / \tau+\sqrt{D x} \xi(t)
$$

Where $\mathrm{b}$ is the immigration rate from a large regional community, $-x / \mathrm{T}$ represents competition for finite resources and $D$ is related to demographic stochasticity; $\xi(t)$ is a Gaussian white noise. This suggests that the neutral assumption could correspond to a scenario in which species originate and become extinct on the same timescales of fluctuations of the whole ecosystem (Gilbert and Lechowicz, 2004; Leigh, 2007).

\section{Ecological Forecasting}

Ecological forecasting is an integrated aspect which utilizes knowledge of ecology, physiology and physics in order to predict how ecosystems will change in the future in response to environmental factors like climate change. The main aim of this approach is providing researchers including resource managers and designers of both freshwater and marine reserves with relevant data that produce information that may be useful in policy formulation, in advance, so as to curtail unwanted future changes. It is a form of adaptation to global warming (Clark, 2001).

Global warming is one of the most important environmental factors impacting organisms today. Most physiological processes are affected by temperature. Changes in weather and climate even in small degree may lead to large changes in the growth, reproduction and survival of animals and plants. The increase in atmospheric greenhouse gases due to human activity caused most of the warming observed during the postindustrial era. These changes negatively affect both humans and the natural ecosystems, thereby disrupting the biodiversity (Keaney, 2006).

The key aspect of ecological forecasting is the ability to predict where, when and with what degree impacts are bound to occur. This is important so that the authorities concerned can avoid the impeding crises or at least set emergency alert for them. Ecological forecasting involves application of existing knowledge of both biotic and abiotic components of the ecosystem, it also assist us in knowing how the changes in environmental factors can lead to changes whether temporary or permanent to the ecosystems and consequently the biodiversity (Clark, 2001; Keaney, 2006). There are two broad approaches to the application of ecological forecasting, these are:

- Climate Envelope Modeling: This type of approach uses statistical correlations within and without existing species distributions and the abiotic components in order to define a species' adaptation to any environmental change. Envelopes of tolerance are then drawn around existing ranges. By predicting future levels of factors such as temperature, $\mathrm{pH}$, rainfall, and salinity, and other variables are then predicted. This method is good for observation of large numbers of species, which are likely not a good means of predicting effects at fine scales (Wethey et al., 2008).

- Niche level modeling: This ecological forecasting approach correlates physiological data and information about a species to models of animal and plant body temperature. Unlike the climate envelope approach, abiotic factors that serve as environmental variables are predicted at the level of the niche and are therefore much more exact. The draw back to this approach is usually more time consuming (Keaney, 2006).

Biodiversity Action Plan (BAP)

A Biodiversity Action Plan (BAP) is an internationally recognized program addressing threatened species and by a continuous (random) variable $x$, whose evolution is governed by the following Langevin equation: habitats and is designed to protect and restore biological systems. The original impetus for these plans derives from the 1992 Convention on Biological Diversity (CBD). As of 2009, 191 countries have ratified the CBD, but only a fraction of these have developed substantive BAP documents (Glowka et al., 1994; Dobson, 2005).

The principal elements of a BAP typically include: (a) preparing inventories of biological information for selected species or habitats; (b) assessing the conservation status of species within specified ecosystems; (c) creation of targets for conservation and restoration; and (d) establishing budgets, timelines and institutional partnerships for implementing the BAP (Dobson, 2005).

A fundamental method of engagement to a BAP is thorough documentation regarding individual species, with emphasis upon the population distribution and conservation status. For many mammal, bird and reptile species, information is often available in published literature; however, for many plant species as well as invertebrate species, such information may require considerable local data collection. It is also useful to compile time trends of population estimates in order to understand the dynamics of population variability and vulnerability. In some parts of the world, complete species inventories are not realistic; for example, in the Madagascar, dry deciduous forests, many species are completely undocumented and much of the region has never even been systematically explored by scientists (Noss, 1990; Dobson, 2005; Anonymous, 2007).

A species plan component of a country's BAP should ideally entail a thorough description of the range, habitat, behaviour, breeding and interaction with other species. Once a determination has been made of conservation status (e.g. rare, endangered, threatened, vulnerable), a plan can then be created to conserve and restore the species population to target levels. Examples of programmatic protection elements are: habitat restoration; protection of habitat from urban development; establishment of property ownership; limitations on grazing or other agricultural encroachment into habitat; reduction of slash and burn agricultural practices; outlawing killing or collecting the species; restrictions on pesticides use; and control of other environmental pollution. The plan should also articulate which public and private agencies should implement the protection strategy and indicate budgets available to execute this strategy (IUCN Red-List Statistics, 2008).

\section{Biodiversity Planning: A New Way of Thinking}

It was first adopted by EU Heads of State at the EU Summit in Gothenburg in June 2001. It was decided that "biodiversity decline should be halted with the aim of reaching this objective by 2010 . The definition of biodiversity under the Convention on Biological Diversity now recognises that biodiversity is a combination of ecosystem structure and function, as much as its components e.g. species, habitats and genetic resources. Article 2 states: 
In addressing the boundless complexity of biological diversity, it has become conventional to think in hierarchical terms, from the genetic material within individual cells, building up through individual organisms, populations, species and communities of species, to the biosphere overall...At the same time, in seeking to make management intervention as efficient as possible, it is essential to take an holistic view of biodiversity and address the interactions that species have with each other and their non-living environment, i.e. to work from an ecological perspective.

The World Summit on Sustainable Development endorsed the objectives of the Convention on Biological Diversity to "achieve by 2010 a significant reduction of the current rate of biodiversity loss at the global, regional and national level as a contribution to poverty alleviation and to the benefit of life on Earth". To achieve this outcome, biodiversity management will depend on maintaining structure and function (Mace and Baillie, 2007).

\section{REFERENCE}

Amarasekare, P. (2004): The role of density-dependant dispersal in source-sink dynamics. Journal of Theoretical Biology, 226: 159-168.

Anonymous (2007): Rising to the climate challenge". Nature $\quad 449 \quad$ (7164): 755. http://www.nature.com/nature/journal/v449/n7 164/full/449755a.html. Retrieved 2007-11-06.

Azaele, S.; Pigolotti, S., Banavar, J. R., and Maritan, A. (2006). "Dynamical evolution of ecosystems". Nature 444 (7121): 926-928.

Bejan, A., Lorente, S. and Lee, J. (2008): Unifying constructal theory of tree roots, canopies and forests, J. Theor. Biol., 254: 529-540.

Bejan, A. and Lorente, S. (2010): The constructal law of design and evolution in nature, Philosophical Transactions of the Royal Society B, 365: 13351347.

Brown, J. H., Gillooly, J. F., Allen, A. P., Savage, V. M., and West, G. B. (2004): "Toward a metabolic theory of ecology". Ecology 85 (7): 1771-89.

Clark, J.S. (2001): Ecological forecasts: an emerging imperative. Science 293: 657-660.

Dobson, A. (2005): Monitoring global rates of biodiversity change: challenges that arise in meeting the Convention on Biological Diversity (CBD) 2010 goals. Philosophical Transactions of The Royal Society B. 360 (1454): 229-241.

Eldredge, N. (1998): Life in the balance: Humanity and the Biodiversity Crisis, Princeton University Press, US, pp:1-50.

Ewens, W. (1972): "The sampling theory of selectively neutral alleles", Theoretical Population Biology, volume 3, pp: 87-112.

Fairbairn, D.J. (1997): "Allometry for Sexual Size Dimorphism: Pattern and Process in the Coevolution of Body Size in Males and Females". Annu. Rev. Ecol. Syst. 28 (1): 659-687. doi:10.1146/annurev.ecolsys.28.1.659

Gallardo, C.S. and Penchaszadeh, P.E. (2001): Hatching mode and latitude in marine gastropods: revisiting Thorson's paradigm in the southern hemisphere. Marine Biology, 138 547-552

\section{CONCLUSION}

Ecosystem models are a development of theoretical ecology which goal is to characterize major ecosystem dynamics in order to predict the behaviors of the systems. Ecosystems that lose biodiversity become more fragile and susceptible to collapse. This is because no species lives independent of other species. All species are interdependent, connected in a web of life that forms the foundation of the ecosystem. With the loss of each species, the possibility of a catastrophic collapse increases. Ecosystem models typically simplify the systems to a limited number of pragmatic components. These can be in form of analyzing a particular species abundance, composition etc or in form of studying species broad functional types such as autotrophs, heterotrophs or saprotrophs. The structure of ecosystem models enables researchers to design and consequently formulate monitoring programs that will be useful to the conservation of biodiversity.

Gilbert, B. and Lechowicz M.J. (2004): "Neutrality, niches, and dispersal in a temperate forest understory". PNAS 101 (20): 7651-7656.

Gillooly, J.F., Allen, A.P., West, G.B., and Brown, J.H. (2005): "The rate of DNA evolution: Effects of body size and temperature on the molecular clock". Proc Natl Acad Sci U S A. 102 (1): 140$5 . \quad$ doi:10.1073/pnas.0407735101. PMID 15618408.

Glowka, L., Françoise B., Hugh S., Jeffrey A. M. and Lothar G. (1994): Guide to the Convention on Biodiversity. IUCN. ISBN 2-8317-0222-4.

Gusev, A.V. (1978): Monogenoidea of freshwater fish. Principles of systematics, analysis of the world fauna and its evolution. Parasitologicheskij Sbornik, 28: 96-198 (in Russian).

Hubbell, S.P. (2001): The Unified Neutral Theory of Biodiversity and Biogeography. Princeton University Press.

Hui, C., McGeoch, M.A., Reyers, B., le Roux, P.C., Greve, M. and Chown, S.L. (2009): Extrapolating population size from the occupancy-abundance relationship and the scaling pattern of occupancy. Ecological Applications, 19, 20382048.

Hutchinson, G., and MacArthur, R. (1959): "A theoretical ecological model of size distributions among species of animals". Am. Nat. 93: 117-125. doi: $10.1086 / 282063$.

Israel G (1988): "On the contribution of Volterra and Lotka to the development of modern biomathematics". History and Philosophy of the Life Sciences 10 (1): 37-49

IUCN Red-list statistics (2006): Government of St. Lucia (2001). "National Biodiversity Strategy and Action Plan of St. Lucia". http://www.slubiodiv.org/The_Project/Informati on/Printed_Materials/NBSAP/nbsap.html. Retrieved 2006-08-30.

Johnson, N.L. Kotz, S. and Balakrishnan, N. (1997): Discrete Multivariate Distributions, Wiley.

Kearney, M. (2006): Habitat, environment and niche: what are we modelling? Oikos 115: 186-191.

Kleiber, M. (1932): "Body size and metabolism". Hilgardia 6: 315-351. 
Kleiber M (1947): "Body size and metabolic rate". Physiological Reviews 27: 511-541

Kozlowski, J. and Konarzewski, M. (2004): "Is West, Brown and Enquist's model of allometric scaling mathematically correct and biologically relevant?". Functional Ecology 18: 283-9

Krug, P.J. (1998): Poecilogony in an estuarine opisthobranch: planktotrophy, lecithotrophy, and mixed clutches in a population of the ascoglossan Alderia modesta. Marine Biology, 132:483-494.

Leigh E.G. (Jr) (2007): "Neutral theory: a historical perspective". Journal of Evolutionary Biology 20 (6): 2075-2091.

Lotka, A.J. (1925): The Elements of Physical Biology, Williams \& Williams co., Baltimore, USA.

Mace, G.M. and Baillie, J.E.M. (2007): The 2010 biodiversity indicators: Challenges for science and policy. Conservation Biology. 21(6): 14061413

Mileikovsky, S. A. (1971): Types of larval development in marine bottom invertebrates, their distribution and ecological significance: a reevaluation. Marine Biology 19: 193-213.

Noss, R.F. (1990): Indicators for Monitoring Biodiversity: A Hierarchical Approach. Conservation Biology 4 (4) $355-364$.

Rapoport, E. H. (1982): Areography. Geographical Strategies of Species. Trad. B. Drausal, Pergamon Press, Oxford.

Reich PB, Tjoelker MG, Machado JL, and Oleksyn J (2006): "Universal scaling of respiratory metabolism, size, and nitrogen in plants". Nature 439 (7075): 457-61.

Rohde, K. (1985): Increased viviparity of marine parasites at high latitudes. Hydrobiologia 127: 197-201.

Rohde, K. (1992): "Latitudinal gradients in speciesdiversity: the search for the primary cause". Oikos 65 (3): 514-527. doi:10.2307/3545569.

Rohde, K., Heap M. and Heap, D. (1993): Rapoport's rule does not apply to marine teleosts and cannot explain latitudinal gradients in species richness. American Naturalist 142: 1-16.

Rohde, K. (1996): Rapoport's Rule is a local phenomenon and cannot explain latitudinal gradients in species diversity. Biodiversity Letters, 3, 10-13.
Savage V.M., Gillooly J.F., Brown J.H., West G.B. and Charnov E.L. (2004): "Effects of body size and temperature on population growth". American Naturalist 163 (3): 429-441.

Savage VM, Allen AP, Brown JH, Gillooly JF, Herman AB, Woodruff WH, West GB (2007): "Scaling of number, size, and metabolic rate of cells with body size in animals". Proc Natl Acad Sci U SA. 104 (11): 4718-23. doi:10.1073/pnas.0611235104. PMID 17360590.

PMC 1838666. http://www.pnas.org/cgi/pmidlookup?view=lon g\&pmid $=17360590$.

Stevens, G. C. (1996): Extending Rapoport's rule to Pacific marine fishes. Journal of Biogeography 23:149-154.

Thorson, G. (1957): Bottom communities (sublittoral or shallow shelf). In "Treatise on Marine Ecology and Palaeoecology" (Ed J.W. Hedgpeth) pp. 461-534. Geological Society of America.

Vallade, M. and Houchmandzadeh, B. (2003): "Analytical solution of a neutral model of biodiversity". Phys. Rev. E 68 (061902): 061902.

Verma, P.S. and Agarwal, V.K. (2007): Environmental Biology: Principles of Ecology. $11^{\text {th }}$ reprinted edition, S.Chand \& Co., Ltd., New Delhi, India. Pp: 3-153.

Volterra, V. (1926): Fluctuations in the abundance of a species considered mathematically. Nature 118: 558-560.

Wethey, D.S, and S.A. Woodin. (2008): Ecological hindcasting of biogeographic responses to climate change in the European intertidal zone. Hydrobiologia 606:139-151.

Wikipedia Encyclopedia (2009): Ecosystem Models. Retrieved from http://en.wikipedia.org/wiki/ecosystem model, Last modified on $4^{\text {th }}$ June, 2009.

Zuckerberg, B., W.F. Porter, and K. Corwin. (2009): The consistency and stability of abundanceoccupancy relationships in large-scale population dynamics. Journal of Animal Ecology, 78: 172-181. 